\title{
The Relationship between Dimensions of Health Related Quality of Life and Health Conditions among Elderly People: A Fuzzy Linear Regression Approach
}

\author{
Lazim Abdullah $^{1} \&$ Noor Jamalina Mohd Jamal ${ }^{1}$ \\ ${ }^{1}$ School of Informatics and Applied Mathematics, Universiti Malaysia Terengganu, Malaysia \\ Correspondence: Lazim Abdullah, School of Informatics and Applied Mathematics, Universiti Malaysia \\ Terengganu, Kuala Terengganu, 21030 Malaysia. Tel: 609-668-3335. E-mail: lazim_m@umt.edu.my
}

Received: April 3, 2015

Accepted: November 1, 2015

Online Published: December 24, 2015

doi:10.5539/mas.v10n2p1

URL: http://dx.doi.org/10.5539/mas.v10n2p1

\begin{abstract}
Health Related Quality of Life (HRQoL) is one of the escalating subjects used for assessing health condition among patients who suffer specific diseases or ailments. It has been known that dimensions of HRQoL are able to mirror one's overall health condition using mainly standard statistical technique. However, devising the extent of contribution of multiple dimensions towards overall health conditions is not straight forward as the arbitrary nature of HRQoL dimensions. Therefore this paper aims to propose a model to explain the relationship between HRQoL dimensions and overall health condition using a matrix driven fuzzy linear regression. An experiment was conducted to measure the strength of the relationship among elderly people via judgment provided by ten decision makers. The health condition linguistic data and scaled data of regularity of experiencing health-related problems among elderly people were given by the decision makers. The five stepwise computations based on matrix-driven fuzzy linear regression were proposed to describe the relationship. It is found that nearly forty six percent variations in overall health condition of elder people were explained by the eights HRQoL dimensions. The employment of matrix-driven multivariate fuzzy linear regression model has successfully identified the strength of the relationship between multi dimensions of HRQoL and overall health condition in the case of elderly people.
\end{abstract}

Keywords: quality of life, fuzzy linear regression, coefficient of determination, decision making, elderly people

\section{Introduction}

\subsection{Background}

Recent developments in economic activities and advanced technologies have heightened the need for creating higher quality of life (QoL) living environment. QoL has been defined in many ways depending on the social status and values that have been practising in a country. QoL also has been extended to health related matters and normally known as health related quality of life (HRQoL). Guyatt et al. (1993) divided HRQoL into two types, generic or specific. Generic HRQoL measures the QoL of well-being in general, while specific HRQoL measures the QoL of well-being by specific condition or disease. Very often, the terms QoL and HRQoL are interchangeably used with little dissimilarity between the two concepts. But a loose distinction between these two concepts can be drawn by giving more emphasis on health related issues rather than the general outlook of quality of life. In many literatures, HRQoL is viewed as a multi-dimensional concept (Philips 2006; Bowling 1995; Schron \& Shumaker, 1992; Wenger et al. 1984). Bowling (1995) defined HRQoL as optimum level of multiple physical roles such as work, career, parent and social functioning, including relationships and perceptions of health, fitness, life satisfaction and well-being. Therefore, the important concept of HRQoL is multi-dimensionality where it could mirror an overall health condition of patients or general group of people. Since HRQoL is related to multi-dimensional concepts, it is therefore imperative to choose a reliable multi-criteria approach in measuring or evaluating these multi-dimensional concepts and observing how HRQoL is impacting human lives. Measurements of HRQoL ideally must consistent with the multi-dimension of HRQoL. There are many researches that discussed measurements of HRQoL. However, most of the researches use the statistical approaches where data are administered from the perspective of the patients using self-completed questionnaires. Examples of such researches can be retrieved from Varni et al. (2007), Hill et al. (2007), and 
Godes, et al. (2013).

\subsection{HRQoL among Elderly}

Research of HRQoL is not only focused on specific groups of patients with specific diseases but also studied according to patients' personal profiles such as genders, age groups and social economic status. Barr et al. (2007) for example, conducted a research of HRQoL among children while Wong et al. (2009) implemented a HRQoL research among adolescents. Another age group that vulnerable to health related problems is elderly people. Many researches have been conducted to measure health related status among elderly people. Cavlak et al. (2009) studied the effects of musculoskeletal pain on HRQoL and examined gender differences among elderly people living in Turkey. Chia et al. (2004) investigated the impact of visual impairment on HRQoL in an older population. There was also a study conducted by Rueda and Artazcoz (2009) where gender inequalities of health status among elderly people were measured. Relationships between elder people and income were studied by Huguet, et al. (2008). They assessed the independent effect of income on HRQoL among older adults in Canada and the United States. Liang and $\mathrm{Wu}$ (2014) explored the HRQoL of empty-nest elderly in rural China with the aim to raise social concern for their HRQoL.

\subsection{Multi-Criteria Evaluation}

Besides the statistical methods, there were other approaches that have been employed in analyzing multi-dimension of HRQoL. These approaches were relied on intangibility, multi-dimensionality and arbitrary definitions of HRQoL. It is proper to approach the arbitrary nature of HRQoL with a fuzzy multi-criteria evaluation. One of the most popular approaches in subjective evaluation is fuzzy multi-criteria decision making. Bellman and Zadeh (1970) and a few years later, Zimmermann (1985) introduced fuzzy sets into multi-criteria decision making. The approaches of fuzzy multi-criteria decision making have been tested successfully in many real life applications, including health sciences and sustainable human well-being. For example, the fuzzy approach has been applied in medical decision making (Reyna 2008; Toress \& Nieto 2006; Phuonga \& Kreinovich 2001) and also in measuring quality of life (Abdullah \& Md Tap 2008; Abdullah \& Md Tap 2009). The method of fuzzy decision making fuzzy simple additive weight was employed by Abdullah and Jamal (2011) to weight dimensions of HRQoL. The same authors proposed a four-step computation of centroid method ranking fuzzy numbers and apply it in ranking multi-dimension of HRQoL (Abdulah \& Jamal 2010). The multi-dimensionality of HRQoL also can be viewed as a problem that involves multiple variables. One of the most popular linear methods in multi-variate analyses is multiple linear regressions. However the arbitrary of HRQoL adds a new breadth in describing the relationship between multi-dimension of HRQoL and overall health condition. The new analysis of multiple linear regressions in HRQoL reckons fuzzy numbers in the regression model and interpretations. The concept of fuzzy numbers was embedded into the predictors and response variables of multiple linear regressions.

\subsection{Motivation and Objectives}

There has been handful of research using fuzzy linear regression to model real life applications. Most of the research of fuzzy linear regression discussed the complexity of mathematical programming involved. Some real life applications can be seen in information technology realm. For example, Kahraman (2002) forecasts sale levels of computer equipment in Turkey using fuzzy linear regressions. Wu et al. (2009) describes an application of the fuzzy linear regression analysis for land-cover classification of Landsat TM data in remote sensing field. Abdullah and Zakaria (2012) applied the fuzzy linear regression model to investigate the relationship between variables impacting car sales volume. Pan et al. (2009) introduced a matrix-driven multivariate fuzzy linear regression as part of their efforts to improve computational efficiency. The method has been successfully tested in engineering study of estimating bridge performance. Researches in sustainable health sciences using this fuzzy linear regression analysis, nevertheless, have been given little attention. Thus, using fuzzy linear regression in health related problem offer a new perspective in application of the relationship analysis. Motivated by the low computational risk of the method and silent attempt of the method in modeling in variables in health related sciences, this paper extends the method to find the relationships between HRQoL dimensions with general health condition among elderly. Differently from the typical statistical approaches where data are directly collected from patients, the method introduces fuzzy numbers to enhance linguistics judgment made by decision makers. Specifically the objective of this paper is to model the relationship between dimensions of HRQoL to overall health condition of elderly people using the matrix-driven multivariate fuzzy linear regression. As the way to appropriately deal with this mathematical modeling, the term dimensions of HRQoL is replaced with predictors and will be used interchangeably throughout the paper. 


\section{Matrix Driven Fuzzy Linear Regression}

For the purpose of clarity and self-explained, part of the method proposed by Pan et al. (2009) is restructured and simplified. The matrix-driven multivariate fuzzy linear regression for HRQoL modeling is given as follows.

If $\widetilde{Y}_{i}$ is triangular fuzzy number, $\left(y_{n},(1-\mu) e_{n, L},(1-\mu) e_{n, R}\right)$ then the method of least square is used to find that particular regression line $\left(\hat{Y}_{i}\right)$ where the sum of squared deviations of the data points $\left(\widetilde{Y}_{i}\right)$ above or below is minimized. To facilitate the fuzzy regression analysis, matrix algebra is employed. The general fuzzy linear model can be expressed in the following matrix form

$$
\tilde{Y}=X \hat{\widetilde{\beta}}
$$

where

$$
\begin{aligned}
& \widetilde{Y}=\left[\begin{array}{c}
\left(y_{1},(1-\mu) e_{1, L},(1-\mu) e_{1, R}\right) \\
\left(y_{2},(1-\mu) e_{2, L},(1-\mu) e_{2, R}\right) \\
\vdots \\
\left(y_{n},(1-\mu) e_{n, L},(1-\mu) e_{n, R}\right)
\end{array}\right] \\
& X=\left[\begin{array}{ccccc}
1 & x_{11} & x_{21} & \cdots & x_{k 1} \\
1 & x_{12} & x_{22} & \cdots & x_{k 2} \\
\vdots & \vdots & \vdots & \vdots & \vdots \\
1 & x_{1 n} & x_{2 n} & \cdots & x_{k n}
\end{array}\right] \\
& \hat{\widetilde{\beta}}=\left[\begin{array}{cccc}
\hat{\widetilde{\beta}}_{0} & \hat{\widetilde{\beta}}_{1} & \vdots & \hat{\widetilde{\beta}}_{k}
\end{array}\right]=\left[\begin{array}{c}
\left(a_{0},(1-\mu) c_{0, L},(1-\mu) c_{0, R}\right) \\
\left(a_{1},(1-\mu) c_{1, L},(1-\mu) c_{1, R}\right) \\
\vdots \\
\left(a_{n},(1-\mu) c_{n, L},(1-\mu) c_{n, R}\right)
\end{array}\right]
\end{aligned}
$$

In the above equations, matrices $Y$ and $\mathcal{A} X$ are the data matrices associated with the response variable and predictor variables, respectively. Matrix $\widetilde{\beta}$ contains the least squares estimates of the regression coefficients. To obtain the regression parameters, Equation (1) can be transformed using equation (5).

$$
\left(X^{\prime} X\right) \beta=X^{\prime} Y
$$

where $X^{\prime}$ is the transpose matrix of $X$.

By matrix operations, the regression coefficients can be derived as follows:

$$
\beta=\left(X^{\prime} X\right)^{-1} X^{\prime} Y
$$

where $\left(X^{\prime} X\right)^{-1}$ is the inverse matrix of $X^{\prime} X$.

The fitted fuzzy regression equation can be developed based on the estimated regression coefficients. After establishing the regression equation, it is good to measure the quality or reliability of the fitted regression equation. The fuzzy coefficient of determination $(H R)^{2}$ is used to interpret the proportion of the total variation in $\mathrm{Y}$ explained by the regression line. The coefficient is defined by

where $Y$ is the mean of fuzzy data $Y$.

$$
(H R)^{2}=\frac{\sum_{i=1}^{n}\left(\hat{Y}_{i}-\overline{\widetilde{Y}}\right)^{2}}{\sum_{i=1}^{n}\left(\widetilde{Y}_{i}-\overline{\widetilde{Y}}\right)^{2}}
$$

Likewise, the fuzzy correlation coefficient $(H R)$ is the square root of $(H R)^{2}$, which can be used to evaluate the 
strength of the linear relationship between predictors and response variables. Fuzzy linear regression equation, coefficient of determination and correlation coefficient are the main modeling measures that would be obtained from the HRQoL experiment. The method is applied to model an experiment of HRQoL's predictors and response variables.

\section{Experiment}

An experiment was conducted to collect linguistic judgment data over the health related status of elderly people. Ten decision makers comprise three medical officers (D1, D2, D3) and seven nurses (D4, D5, D6, D7, D8, D9, D10) were voluntarily formed a group of decision makers. All the decision makers were attached to a Malaysian public hospital. Decision makers were asked to provide judgment over related problem among elderly people using an approach of guided interview. The closed ended questions were developed by the authors based on literature in health related quality of life and also the SF-36 questionnaire (Ware, 1993). There were eight dimensions in the questionnaire and decision makers make their judgment with respect to the eight health related dimensions among elderly. There are three to seven items for every dimension. The eight dimensions are predictors $\left(x_{1}, \ldots, x_{8}\right)$ and the response variable is overall health condition $\left(Y_{i}\right)$.

The first predictor $x_{1}$ refers to physical aspect in which it focuses more of the outer physical of human body as the general definition. Second predictor, $x_{2}$ refers to role-physical factor, focusing more in measuring the physical functioning of the human himself, whether or not it gives expected response to the daily activities. The third predictor $x_{3}$ is bodily pain. The fourth predictor $x_{4}$ is vitality. The fifth predictor, $x_{5}$ is social aspect where defines more about their social life due to the health condition that they may have. The sixth predictor, $x_{6}$ emotional aspects and measures their emotional side that usually give the major impact to the quality of life of human beings. The seventh predictor, $x_{7}$ is role-emotional defines and measures the functions of mind and emotions due to their health status. The last predictor $x_{8}$ is school/workplace aspect because the major activities that people usually do is schooling or working and this factor is of high importance to be measured because the environment of the schools and workplaces or worship places do give predominant effect to the people due to their health condition.

The decision makers need to respond in five linguistic scales 'never have a problem' (1), 'almost never have problem' (2), 'often have a problem' (3), 'sometimes have a problem' (4), and 'always have a problem' (5) to indicate their views over experience of health related symptoms of the eight predictors among elderly people. Also the decision makers need to answer the overall health condition among elderly people in three linguistic terms 'Good', 'Medium' and 'Low'. Each linguistic term is assigned with a specific fuzzy number to reflect fuzziness of the terms. Linguistic scales and their respective normal triangular fuzzy numbers are given in Table 1.

Table 1. Linguistic terms and fuzzy numbers

\begin{tabular}{lc}
\hline Linguistic term & Triangular Fuzzy Number \\
\hline Good $(\mathrm{G})$ & $(60,80,90)$ \\
Medium $(\mathrm{M})$ & $(40,55,60)$ \\
Low $(\mathrm{L})$ & $(10,20,40)$ \\
\hline
\end{tabular}

The experiment requires two states in the computational process. The first state is rating where the decision makers express their opinions (or performance ratings) with respect to elderly quality of life dimensions and also overall health condition via a guided interview. These ratings are fuzzy data that can be in linguistic terms or verbal assessment. This state aims to convert linguistic terms to fuzzy numbers. In other words, this state ensures all linguistic data are converted into fuzzy numbers prior to be fed into the second state. The second state is relationship state where the relationships between response variable and predictors are accomplished. Validation of the relationship using coefficient of determination and correlation coefficient is also obtained in this state. In short, rating and relationship states are a prerequisite processes in obtaining fuzzy linear regression equation for HRQoL. The flow of the experiment can be depicted in Figure 1. 


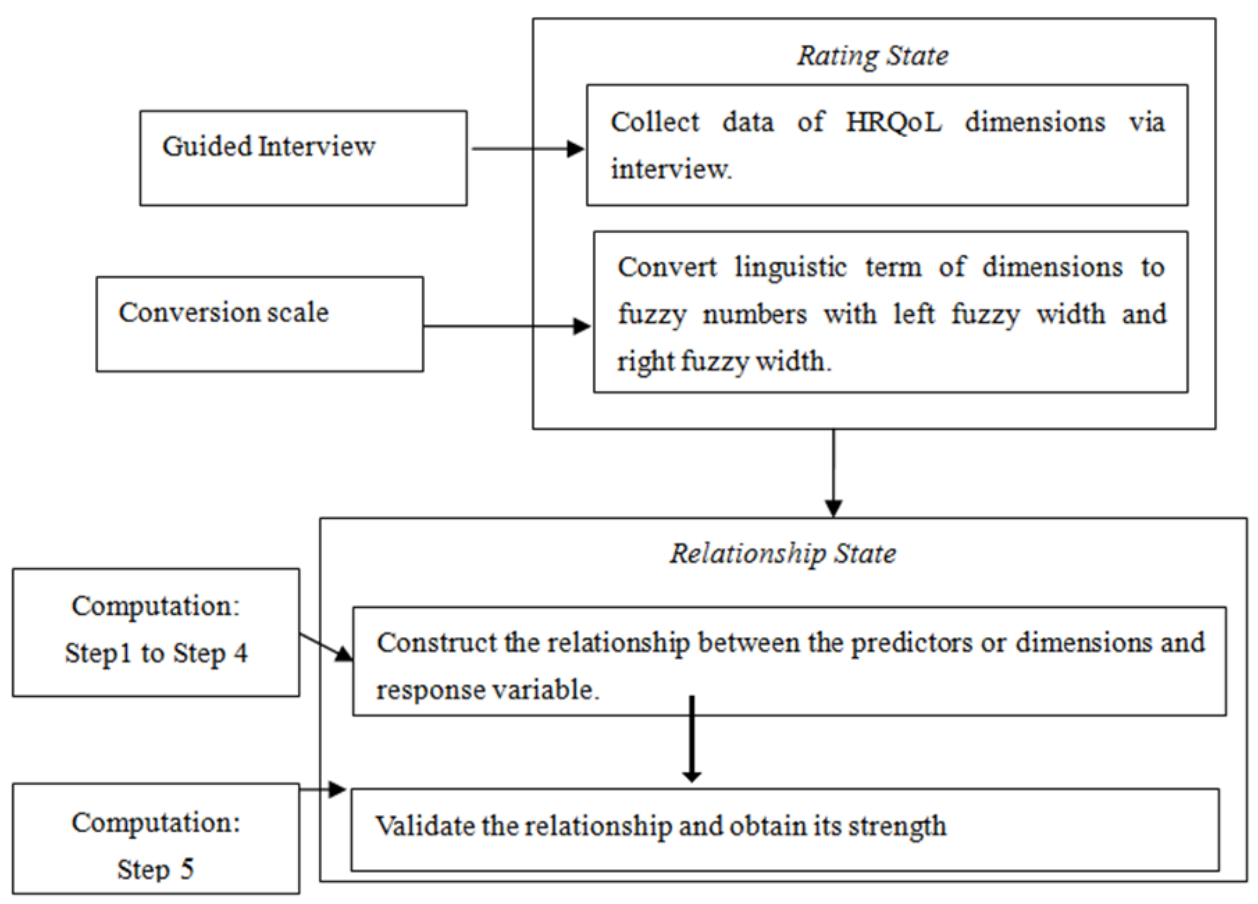

Figure 1. Conceptual framework of the experiment

The rating and relationship states are presented in the following sections to illustrate the whole idea behind the experiment.

\section{Computational Procedures and Results}

Data from the experiment are computed into two states. This section presents the computational procedures for the two states prior to obtaining the relational results.

\section{Rating State}

The method proposed by Pan et al. (2009) is applied to model the experiment of HRQoL. Data obtained from the guided interview were transformed into a ten-by- nine matrix, $X$. Ten represents the number of decision makers and nine represents the predictor and response variable reckoned in this experiment. Table 2 presents the averaged score or rating for each predictor given by decision makers.

Table 2. Averaged score for the predictors given by decision makers

\begin{tabular}{ccccccccc}
\hline Decision makers & $x_{1}$ & $x_{2}$ & $x_{3}$ & $x_{4}$ & $x_{5}$ & $x_{6}$ & $x_{7}$ & $x_{8}$ \\
\hline D1 & 2.4444 & 2.0000 & 3.5000 & 2.1111 & 1.8888 & 1.2857 & 2.1429 & 2.0000 \\
D2 & 2.4350 & 1.8990 & 2.5500 & 1.9988 & 2.3333 & 1.3587 & 1.9999 & 2.0000 \\
D3 & 3.3333 & 3.0000 & 3.5000 & 2.2211 & 1.9888 & 1.2857 & 2.3770 & 3.5000 \\
D4 & 2.4444 & 2.0000 & 3.5000 & 1.9988 & 1.8888 & 1.2857 & 2.1429 & 2.0000 \\
D5 & 3.5555 & 2.0000 & 2.0000 & 2.3343 & 2.5555 & 2.1111 & 2.2333 & 2.0000 \\
\hline D6 & 3.7000 & 3.5000 & 3.5000 & 2.1111 & 1.8888 & 1.3587 & 1.9999 & 1.1999 \\
D7 & 2.4444 & 2.0000 & 2.5500 & 1.9988 & 1.8888 & 1.2857 & 2.1429 & 2.0000 \\
D8 & 2.3344 & 1.9999 & 3.4567 & 3.2222 & 2.5454 & 1.9999 & 2.1122 & 2.0000 \\
D9 & 3.1212 & 3.0000 & 3.5000 & 2.1111 & 1.8888 & 1.2857 & 2.1429 & 2.0000 \\
D10 & 2.4444 & 2.0000 & 3.5000 & 2.1111 & 1.8888 & 1.1222 & 2.1429 & 2.5000 \\
\hline
\end{tabular}

Besides the eight predictors, the experiment also required the overall health condition (response variable) in linguistic terms. The decision makers suggested the overall health condition among elderly people in three linguistic conditions 'good' (G), 'medium' (M) and 'weak' (W). Responses from decision makers were given in linguistic terms and assigned in its respective triangular fuzzy number. The overall health condition for response variable is given in Table 3 . 
Table 3. Linguistic decision and its fuzzy number

\begin{tabular}{cccc}
\hline $\begin{array}{c}\text { Overall Health Condition } \\
\left(\widetilde{Y}_{i}\right) \text { in linguistic term }\end{array}$ & $Y_{i}$ & $e_{L}$ & $e_{R}$ \\
\hline $\mathrm{G}$ & 80 & 20 & 10 \\
$\mathrm{G}$ & 80 & 20 & 10 \\
$\mathrm{G}$ & 80 & 20 & 10 \\
$\mathrm{M}$ & 55 & 15 & 15 \\
$\mathrm{M}$ & 55 & 15 & 15 \\
$\mathrm{M}$ & 55 & 15 & 15 \\
$\mathrm{G}$ & 80 & 20 & 10 \\
$\mathrm{M}$ & 55 & 15 & 15 \\
$\mathrm{G}$ & 80 & 20 & 10 \\
$\mathrm{G}$ & 80 & 20 & 10 \\
\hline
\end{tabular}

The data provided by decision makers in the experiment were entered into the proposed method. Entries of the matrix $X$ represented the averaged scale evaluation for each dimension given by decision makers.

\section{Relationship state}

The vital part of this conceptual model is examining how computation is being carried out in relationship state. Procedures of computations are summarized into five steps and presented as follows:

Step 1: Arrange the predictors in matrix form.

Data from decision makers were arranged as entries of matrix $X$ (refer to equation (3)).

$$
X=\left[\begin{array}{ccccccccc}
1 & 2.4444 & 2 & 3.5 & 2.1111 & 1.8888 & 1.2857 & 2.1429 & 2 \\
1 & 2.4350 & 1.8990 & 2.55 & 1.9988 & 2.3333 & 1.3587 & 1.9999 & 2 \\
1 & 3.3333 & 3 & 3.5 & 2.2211 & 1.9888 & 1.2857 & 2.3770 & 3.5 \\
1 & 2.4444 & 2 & 3.5 & 1.9988 & 1.8888 & 1.2857 & 2.1429 & 2 \\
1 & 3.5555 & 2 & 2 & 2.3343 & 2.5555 & 2.1111 & 2.2333 & 2 \\
1 & 3.7 & 3.5 & 3.5 & 2.1111 & 1.8888 & 1.3587 & 1.9999 & 1.1999 \\
1 & 2.4444 & 2 & 2.55 & 1.9988 & 1.8888 & 1.2857 & 2.1429 & 2 \\
1 & 2.3344 & 1.9999 & 3.4567 & 3.2222 & 2.5454 & 1.9999 & 2.1122 & 2 \\
1 & 3.1212 & 3 & 3.5 & 2.1111 & 1.8888 & 1.2857 & 2.1429 & 2 \\
1 & 2.4444 & 2 & 3.5 & 2.1111 & 1.8888 & 1.1222 & 2.1429 & 2
\end{array}\right]
$$

Step 2: Arrange the response variable in matrix form.

In order to fit in the model, response variable, $Y$, was obtained by considering $\mu=0.5$. Using the equation (2), matrix for $Y$ is given as 


$$
\begin{gathered}
Y=\left[\begin{array}{ccc}
80 & 10 & 5 \\
80 & 10 & 5 \\
80 & 10 & 05 \\
55 & 7.5 & 7.5 \\
55 & 7.5 & 7.5 \\
55 & 7.5 & 7.5 \\
80 & 10 & 5 \\
55 & 7.5 & 7.5 \\
80 & 7.5 & 7.5 \\
80 & 10 & 5
\end{array}\right] \\
\tilde{\beta}=\left[\begin{array}{c}
(71.2774,9.1277,5.8723) \\
(-46.1348,-4.6135,4.6135) \\
(23.5262,2.3526,-2.3526) \\
(7.5280,0.7528,-0.7528) \\
(-62.0578,-6.2058,6.2058) \\
(-0.0019,-0.0002,0.0002) \\
(52.9119,5.2912,-5.2912) \\
(46.2896,4.6290,-4.6290) \\
(7.4316,0.7432,-0.7432)
\end{array}\right]
\end{gathered}
$$

Step 3: Obtain fuzzy regression coefficients.

Matrix of regression coefficients, $\bar{\beta}$ at $\mu=0.5$ can be estimated using equation (5) and (6). The fuzzy regression coefficients is obtained as

The coefficients are fuzzy numbers that consider right and left errors (refer to equation (4)).

Step 4: Obtain fuzzy regression equation.

Overall condition of HRQoL was linearly related to decision makers' opinion on the eight predictors. Therefore, the estimated multiple fuzzy linear regression equation for this relationship is modeled as

$$
\begin{gathered}
\tilde{Y}=(71.27749 .1277,5.8723)+(-46.1348-4.6135,4.6135) x_{1}+(23.5262,2.3526,-2.3526) x_{2} \\
+(7.5280,0.7528,-0.7528) x_{3}+(-62.0578,-6.2058,6.2058) x_{4}+(-0.0019,-0.0002,0.0002) x_{5} \\
+(52.9119,5.2912,-5.2912) x_{6}+(46.2896,4.6290,-4.6290) x_{7}+(7.4316,0.7432,-0.7432) x_{8}
\end{gathered}
$$

From the above equation, it can be seen that the slope of $x_{1}$ is $(-46.1348,-4.6135,4.6135)$, indicating that regardless of other independent predictors, an estimated decrease of 46.1348 in the overall health condition $(\widetilde{Y})$, decrease of 4.6135 in the lower bound (left interval) and an increase of 4.6135 in the upper bound (right interval) occurs for each additional physical aspect $\left(x_{1}\right)$. The estimated slope coefficient of $x_{2},(23.5262,2.3526,-2.3526)$, signifies that regardless of other predictors and for each additional $x_{2}, \widetilde{Y}$ increases by 23.5262 , with an increment of 2.3526 and a decrease of 2.3526 . The estimated slope for $x_{3}$ is $(7.5280,0.7528,-0.7528)$. From this estimation, the increase of 7.5280 in $\widetilde{Y}$ indicates the increases of 0.7528 in the left interval and decreases of 0.7528 in the right interval for each additional of $x_{3}$.

Likewise, the estimated slope for $x_{4}$ is $(-62.0578,-6.2058,6.2058)$, an estimated decrease of 62.0578 in the overall health condition $(\widetilde{Y})$, decreases of 6.2058 in the lower bound and increases with the same value of 6.2058 in the upper bound for each additional in vitality $\left(x_{4}\right)$. Similarly, the estimated slope coefficient of $x_{5}$ is $(-0.0019$, -0.0002. 0.0002) indicating that the decreases of 0.0019 in the $\widetilde{Y}$, a decrease of 0.0002 in left interval and an 
increase 0.0002 in the right interval for each additional in social aspect $\left(x_{5}\right)$. For $x_{6}$, the estimated slope is (52.9119, $5.2912,-5.2912$ ), indicating that regardless of other predictors, an estimated increase in 52.9119 in the overall health condition $(\widetilde{Y})$, with an increase of 5.2912 in the lower bound and a decrease of 5.2912 in the upper bound for each additional in emotional aspect $\left(x_{6}\right)$. For role-emotional aspect, $\left(x_{7}\right)$, the estimated slope is $(46.2896,4.6290$, $-4.6290)$ indicating that for every addition in role-emotional aspect, the overall health condition $(\widetilde{Y})$, increases for 46.2896, also with an increase of 4.6290 for lower bound and a decrease of 4.6290 for upper bound. For the school/workplace aspect $\left(x_{8}\right)$, the estimated slope is $(7.4316,0.7432,-0.7432)$. This estimation shows that regardless for any other predictors, the overall health condition $(\widetilde{Y})$, had an increase of 7.4316 , with a decrease of 0.7432 in upper bound (right interval) and an increase of 0.7432 in lower bound (left interval) for each addition of school/workplace $\left(x_{8}\right)$.

Step 5: Obtain coefficient of determination.

As to observe the contributions of predictors to response variable, coefficient of determination was sought. By substituting $\mu=0.5$ into the Equation (7), the coefficient of determination, $(H R)^{2}$ was obtained. Thus $(H R)^{2}=$ 0.463935565 .

The coefficient shows that forty six percent of the total variation in response dimension $(\widetilde{Y})$ can be explained by the regression line relating to the predictor dimensions. In other words, forty six percent variations in overall health condition of elderly were explained by the eights HRQoL dimensions. The fuzzy correlation coefficient, $H R$ (square root of $(H R)^{2}$ ) thus yields by 0.6811 , signifying a positive linear correlation between the overall health condition and the dimensions.

Summarily, the relationship between HRQoL dimensions and overall health condition found that every dimension contributes differently toward the overall health condition. The dimension of vitality recorded the highest negative contributor toward overall health condition among elderly people while social life dimension has no effect to health condition. The dimension of emotional aspect shows the highest positive contributor toward overall health condition. Taken as a whole, the eight dimensions contribute moderately toward overall health condition in this experiment.

\section{Conclusions}

There are many approaches that have been explored to describe the importance of measurement of multi dimensions of health related quality of life among patient cohorts as it provides a clue to quality of life. However, few have included the arbitrary nature of health related quality of life dimensions in measurement. Further, the strength of relationship between the dimensions and overall health condition from the perspective of decision makers remains untapped. This study aims to integrate the fuzzy data of health related quality of life and decision making approach with predictive multi-variate analysis of linear regression model. The matrix-driven fuzzy linear regression was employed to observe the effect of quality of life dimensions to the overall health condition of elderly people from the perspective of decision makers. Rating state and relationship state were used to analyze the fuzzy data prior to deciding the cause and effect results. The results show that dimension of social life did not contribute to overall health condition. All the other dimensions contributed either negatively or positively to overall health condition. While all individual dimensions contributed differently, only forty six percent of variations in overall health condition were explained by the eight dimensions. The results indicate that the effect of the eight dimensions toward health condition of elderly people from decision makers' point of view is moderate. The application of fuzzy multiple linear regressions in decision making environment is a new approach to model health related quality of life. Since the decisions were made by the selected decision makers thus the results could not be generalized to other case study. However, it is sufficient to provide a novel evidence for the application of matrix-driven fuzzy linear regression to health related quality of life realm.

\section{References}

Abdullah, L., \& Jamal, N. J. (2010). Ranking Fuzzy Numbers and Its Application Health Related Quality of Life Indicators. International Journal of Computer Science and Engineering, 2(8), 2773-2777. Retrieved from 
http://www.arpapress.com/Volumes/Vol11Issue2/IJRRAS_11_2_12.pdf

Abdullah, L., \& Jamal, N. J. (2011). Weight determination of health related quality of life indicators using fuzzy decision making approach. http://dx.doi.org/10.1007/s11482-010-9133-3

Abdullah, L., \& Md Tap, A. O. (2008). Quality of Life Index of Three Selected States in the Peninsular Malaysia: Fuzzy Sets Approach. International Journal of Ecology Economics and Statistics, 11(08), 90-99.

Abdullah, L., \& Md Tap, A. O. (2009). A New Malaysian Quality of Life Index Based on Fuzzy Sets and Hierarchical Needs. http://dx.doi.org/10.1007/s11205-009-9445-6

Abdullah, L., \& Zakaria, N. (2012). Matrix driven multi-variate fuzzy linear regression model in car sales. Journal of Applied Sciences, 12(1), 56-63. http://dx.doi.org/10.3923/jas.2012.56.63

Barr, L., Thibeault, S. L., Muntz, H., \& De Serres, L. (2007). Quality of life in children with velopharyngeal insufficiency. Archives of Otolaryngology, Head \& Neck Surgery, 133(3), 224-229. http://dx.doi.org/10.1001/archotol.133.3.224

Bellman, R., \& Zadeh, L. A. (1970). Decision-making in a fuzzy environment. Management Science, 17, 141-164. http://dx.doi.org/10.1287/mnsc.17.4.B141

Bowling, A. (1995). What things are important in people's lives? A survey of the public's judgments to 363 inform scales of health-related quality of life. Social Science and Medicine, 41(10), 1447-1462. http://dx.doi.org/ 10.1016/0277-9536(95)00113-L

Cavlak, U., Yagci N., Aslan, U. B., \& Ekici, G. (2009). A new tool measuring health-related quality of life (HRQoL): The effects of musculoskeletal pain in a group of older Turkish people. Archives of Gerontology and Geriatrics, 49, 298-303. http://dx.doi.org/10.1016/j.archger.2008.11.002

Chia, E. M., Wang, J. J., Rochtchina, E., Smith, W., Cumming, R. R., \& Mithel, P. (2004). Impact of Bilateral Visual Impairment on Health-Related Quality of Life: The Blue Mountains Eye Study. Investigative Ophthalmology and Visual Science, 45, 71-76. http://dx.doi.org/10.3109/09286580902863072

Godes, J. F., Balic, M. G., \& Sanromà, J. C. (2013) Health-Related Quality of Life Measures for Physically Active Elderly in Community Exercise Programs in Catalonia: Comparative Analysis with Sedentary People. Current Gerontology and Geriatrics Research, 6. http://dx.doi.org/10.1155/2013/168482

Guyatt, G. H., Feeny, D. H., \& Patrick, D. L. (1993). Measuring Health Related Quality of Life. Annals of Internal Medicine, 118(8), 622-629.

Hill, C. D., Edwards, M. C., Thiessen, D., Langer, M. M., Wirth, R. J., \& Burnwinkle, T. M. (2007). Practical Issues in the Applications of Item Response Theory: A Demonstration Using Items From the Pediatric Quality of Life Inventory (PedsQLTM) 4.0 Generic Core Scales. Medical Care, 45(5), 39-47.

Huguet, N., Kaplan, M. S., \& Feeny, D. (2008). Socioeconomic status and health-related quality of life among elderly people: Results from the Joint Canada/United States Survey of Health star. Social Science and Medicine, 66, 803-810.

Kahraman, C. (2002). An application of fuzzy linear regression to the information technology in Turkey. International Journal of Technology Management, 24(2/3), 330-339. http://dx.doi.org/10.1504/IJTM.2002.003059

Liang Y., Wu, W. (2014). Exploratory analysis of health-related quality of life among the empty-nest elderly in rural China: An empirical study in three economically developed cities in eastern China. Health and Quality of Life Outcomes, 12(1), 59. Retrieved from http://www.hqlo.com/content/12/1/59

Pan, N. F., Lin, T. C., \& Pan, N. H. (2009). Estimating Bridge Performance Based On A Matrix-Driven Fuzzy Linear Regression Model. Automation in Construction, 18, 578-586. http://dx.doi.org/10.1016/j.cam.2015.08.009

Phillips, D. (2006). Quality of life: Concept, Policy and Practice. Oxon: Routledge.

Phuonga, N. H., \& Kreinovich, V. (2001). Fuzzy logic and its application in medicine. International Journal of Medical Informatics, 6(2), 165-173. http://dx.doi.org/10.1016/S1386-5056(01)00160-5

Reyna, V. F. (2008). A Theory of Medical Decision Making and Health: Fuzzy Trace Theory. Medical Decision Making, 28(6), 850-865. http://dx.doi.org/10.1177/0272989X08327066

Rueda, S., \& Artazcoz, L. (2009). Gender inequality in health among elderly people in a combined framework of socioeconomic position, family characteristics and social support. Ageing and Society, 29, 625-647. 
http://dx.doi.org/10.1017/S0144686X08008349

Schron, E. B., \& Shumaker, S. A. (1992). The integration of health quality of life in clinical research: experiences from cardiovascular clinical trials. Progress in cardiovascular nursing, 7(1), 21-28.

Sharma, D. (2004). Health-related quality of life and its assessment in GI Surgery. Indian Journal of Surgery, 66, 323-325.

Torres, A., \& Nieto, J. J. (2006). Fuzzy Logic in Medicine and Bioinformatics. Journal of Biomedical and Biotechnology, 1-7. http://dx.doi.org/10.1155/JBB/2006/91908

Varni, J. W., Christine, A. L., \& Tasha, M. B. (2007). Impaired health-related quality of life in children and adolescents with chronic conditions: a comparative analysis of 10 disease clusters and 33 disease categories/severities utilizing the PedsQL ${ }^{\mathrm{TM}} 4.0$ Generic Core Scales. Health Quality of Life Outcome, 5, 43. http://dx.doi.org/10.1186/1477-7525-5-43

Ware, J. E., Jr, Kristin, K. S., \& Kosinski, M. (1993). SF-36 Health Survey Manual and Interpretation Guide. Boston, Massachusetts: Nimrod Press.

Wong, H. B., Machin, D., Tan, S. B., Wong, T. Y., \& Saw, S. M. (2009). Visual Impairment and Its Impact to Health Related Quality of Life in Adolescents. American Journal of Ophthalmology, 147(3), 505-511. http://dx.doi.org/ 10.1016/j.ajo.2008.09.025

Zimmermann, H. J. (1985). Fuzzy Set Theory and Its Applications. Dordrecht, Boston.

\section{Copyrights}

Copyright for this article is retained by the author(s), with first publication rights granted to the journal.

This is an open-access article distributed under the terms and conditions of the Creative Commons Attribution license (http://creativecommons.org/licenses/by/3.0/). 